\begin{tabular}{|c|c|c|c|}
\hline Gene no. (gene name) & Fold change & & Description of proposed function \\
\hline & $\begin{array}{c}\text { Genes with increased relative transcript levels in Ip_2991 deletion } \\
\text { mutant compared to WCFS1 }\end{array}$ & FDR & \\
\hline \multicolumn{4}{|l|}{ Cell envelope } \\
\hline Ip_2988 (zmp3) & 2.2 & $2.70 \mathrm{E}-05$ & extracellular zinc metalloproteinase, M10 family (putative) \\
\hline Ip_2809 & 2.5 & $9.89 \mathrm{E}-03$ & extracellular protein (putative) \\
\hline Ip_0730 (tagO) & 4.4 & $3.37 \mathrm{E}-05$ & undecaprenyl-phosphate N-acetyl-glucosaminyl transferase \\
\hline Ip_2989 (gtcA3) & 45 & $1.83 \mathrm{E}-07$ & teichoic acid glycosylation protein (putative) \\
\hline \multicolumn{4}{|l|}{ Cellular processes } \\
\hline Ip_0214 & 3.1 & $6.66 \mathrm{E}-03$ & chromosome condensation protein (putative) \\
\hline \multicolumn{4}{|l|}{$\begin{array}{l}\text { Central intermediary } \\
\text { metabolism }\end{array}$} \\
\hline IP_1173 & 5.6 & 1.19E-04 & UDP-N-acetylglucosamine 2-epimerase \\
\hline Ip_0181 (mapB) & 5.9 & $3.76 \mathrm{E}-02$ & maltose phosphorylase \\
\hline \multicolumn{4}{|l|}{ Energy metabolism } \\
\hline Ip_2659 (xpkA) & 2.1 & $3.31 \mathrm{E}-04$ & xylulose-5-P phosphoketolase \& fructose-6-P phosphoketolase \\
\hline Ip_3490 & 8.6 & $1.58 \mathrm{E}-02$ & FMN-binding protein \\
\hline \multicolumn{4}{|l|}{ Hypothetical proteins } \\
\hline IP_1726 & 2.1 & $2.97 \mathrm{E}-02$ & unknown \\
\hline Ip_0311 & 2.2 & $1.67 \mathrm{E}-02$ & acetyltransferase (putative) \\
\hline Ip_2066 & 2.2 & $3.76 \mathrm{E}-02$ & unknown \\
\hline Ip_3346 & 2.9 & $2.87 \mathrm{E}-02$ & unknown \\
\hline |p_3002 & 3.2 & $3.18 \mathrm{E}-03$ & integral membrane protein \\
\hline Ip_2230 & 16.7 & $1.70 \mathrm{E}-07$ & unknown \\
\hline Ip_2093 & 18.1 & $1.22 \mathrm{E}-02$ & unknown \\
\hline
\end{tabular}




\begin{tabular}{|c|c|c|c|}
\hline Ip_3348 & 19.1 & $1.41 \mathrm{E}-06$ & unknown \\
\hline \multicolumn{4}{|l|}{ Protein synthesis } \\
\hline Ip_2807 (tyrS) & 2.8 & $3.83 \mathrm{E}-02$ & tyrosine--tRNA ligase \\
\hline \multicolumn{4}{|c|}{ Purines, pyrimidines, nucleosides and nucleotides } \\
\hline Ip_2702 (pyrC) & 4.1 & $3.21 \mathrm{E}-03$ & dihydroorotase \\
\hline Ip_2699 (pyrD) & 4.9 & $4.28 \mathrm{E}-04$ & dihydroorotate oxidase \\
\hline Ip_2701 (pyrAA) & 5.6 & $1.00 \mathrm{E}-04$ & carbamoyl-phosphate synthase, pyrimidine-specific, small chain \\
\hline Ip_2700 (pyrAB) & 6.8 & $2.71 \mathrm{E}-05$ & carbamoyl-phosphate synthase, pyrimidine-specific, large chain \\
\hline Ip_2698 (pyrF) & 7 & $1.03 E-05$ & orotidine-5'-phosphate decarboxylase \\
\hline Ip_2697 (pyrE) & 17 & $1.76 \mathrm{E}-07$ & orotate phosphoribosyltransferase \\
\hline \multicolumn{4}{|c|}{ Regulatory functions } \\
\hline Ip_1938 & 2.2 & $2.80 \mathrm{E}-02$ & transcription regulator, LysR family \\
\hline Ip_2704 (purR1) & 2.6 & $2.71 \mathrm{E}-05$ & pyrimidine operon regulator \\
\hline \multicolumn{4}{|c|}{ Transport and binding proteins } \\
\hline Ip_2992 (mntH2) & 2.4 & $1.23 E-02$ & manganese transport protein \\
\hline Ip_0092 & 2.5 & $3.46 \mathrm{E}-03$ & ABC transporter, substrate binding protein, oligopeptide \\
\hline Ip_2371 (pyrP) & 2.6 & $9.81 \mathrm{E}-07$ & uracil transport protein \\
\hline Ip_0286 (pts6C) & 5.1 & $3.39 \mathrm{E}-03$ & cellobiose PTS, EIIC \\
\hline Ip_1792 & 19.7 & $1.43 \mathrm{E}-02$ & $A B C$ transporter, permease protein \\
\hline Ip_p3_38 & 3.2 & $1.10 \mathrm{E}-03$ & nickase \\
\hline
\end{tabular}


Genes with decreased relative transcript levels in Ip_2991 deletion mutant compared to WCFS1

Biosynthesis of cofactors, prosthetic groups, and carriers

Ip_0369 (gshR1)

glutathione reductase

Cellular processes

Ip_2210 (ftsK2)

cell division protein FtsK

\section{DNA metabolism}

Ip_1839 (parC)

$1.51 \mathrm{E}-05$

topoisomerase IV, subunit A

\section{Energy metabolism}

Ip_3595 (rhaB)

Ip_3449 (nox5)

\section{Hypothetical proteins}

Ip_1533

Ip_0753

Ip_0967

Ip_2058

Ip_1390

Ip_1136

Ip_2114

Protein synthesis

Ip_0443 (dus1)

3.32E-07 rhamnulokinase

4.42E-02 NADH oxidase

3.79E-05 methyltransferase (putative)

2.71E-04 integral membrane protein

2.19E-02 unknown

8.25E-03 endonuclease (putative)

4.40E-03 acetyltransferase, GNAT family (putative)

1.45E-04 oxidoreductase, $\operatorname{NAD}(\mathrm{P})$-dependent

1.07E-03 NTP pyrophosphohydrolase (putative)

9.88E-04 tRNA-dihydrouridine synthase 


\section{Regulatory functions}

Ip_0188 (scrR)

Ip_0319

Transport and binding proteins

Ip_2531 (pts18CBA)

Ip_3686

Ip_0317 (potB)

Ip_0218

Ip_2352

Ip_3279 (kup2)

Ip 2351

Ip_0367 (choS)

Ip_0368 (choQ)
$1.12 \mathrm{E}-03$

4.29E-02

9.98E-04

$1.21 \mathrm{E}-03$

$3.12 \mathrm{E}-02$

1.89E-03

$5.98 \mathrm{E}-06$

$6.55 \mathrm{E}-03$

$3.26 \mathrm{E}-03$

3.29E-05 oligosucrose operon repressor

transcription regulator, spermidine/putrescine transport operon

$\mathrm{N}$-acetylglucosamine and glucose PTS, EIICBA

$A B C$ transporter, substrate binding protein

spermidine/putrescine $A B C$ transporter, permease protein

$A B C$ transporter, ATP-binding protein

$A B C$ transporter, ATP binding binding protein, D-Methionine -like precursor potassium uptake protein

$A B C$ transporter, permease protein D-Methionine -like precursor

glycine betaine/carnitine/choline $\mathrm{ABC}$ transporter, substrate binding and permease protein

glycine betaine/carnitine/choline ABC transporter, ATP-binding protein 